\title{
Article \\ Antimicrobial Resistance in Salmonella Isolated from Food Workers and Chicken Products in Japan
}

\author{
Yoshimasa Sasaki ${ }^{1,2, *}$, Hiromi Kakizawa ${ }^{3}$, Youichi Baba ${ }^{3}$, Takeshi Ito ${ }^{3}$, Yukari Haremaki ${ }^{4}$, Masaru Yonemichi ${ }^{4}$, \\ Tetsuya Ikeda ${ }^{5}$, Makoto Kuroda ${ }^{6}$, Kenji Ohya ${ }^{7}$, , Yukiko Hara-Kudo ${ }^{7}$, Tetsuo Asai ${ }^{2}{ }^{\circledR}$ and Hiroshi Asakura ${ }^{1,2}$ (D) \\ 1 Division of Biomedical Food Research, National Institute of Health Sciences, 3-25-26, Tonomachi, \\ Kawasaki-ku, Kawasaki 210-9501, Kanagawa, Japan; hasakura@nihs.go.jp \\ 2 Department of Applied Veterinary Science, The United Graduate School of Veterinary Science, \\ Gifu University, 1-1, Yanagido, Gifu 501-1193, Gifu, Japan; tasai@gifu-u.ac.jp \\ 3 Incorporated Foundation Tokyo Kenbikyo-in, 1-100-38 Takamatsu-cho, Tachikawa 190-0011, Tokyo, Japan; \\ kakizawa@kenko-kenbi.or.jp (H.K.); baba@kenko-kenbi.or.jp (Y.B.); tito@kenko-kenbi.or.jp (T.I.) \\ 4 BML Food Science Solutions, Inc., 1549-7, Matoba, Kawagoe 350-1101, Saitama, Japan; \\ yharemak@bml.co.jp (Y.H.); m-yonemi@bml.co.jp (M.Y.) \\ 5 Department of Infectious Diseases, Hokkaido Institute of Public Health, Kita19 Nishi 12, Kita-ku, \\ Sapporo 060-0819, Hokkaido, Japan; ikeda@iph.pref.hokkaido.jp \\ 6 Pathogen Genomics Center, National Institute of Infectious Diseases, 1-23-1 Toyama, Shinjuku-ku, \\ Tokyo 162-8640, Japan; makokuro@niid.go.jp \\ 7 Division of Microbiology, National Institute of Health Sciences, 3-25-26, Tonomachi, Kawasaki-ku, \\ Kawasaki 210-9501, Kanagawa, Japan; kohya@nihs.go.jp (K.O.); ykudo@nihs.go.jp (Y.H.-K.) \\ check for \\ * Correspondence: ysasaki@nihs.go.jp; Tel.: +81-44-270-6566; Fax: +81-44-270-6569
} updates

Citation: Sasaki, Y.; Kakizawa, H.; Baba, Y.; Ito, T.; Haremaki, Y.; Yonemichi, M.; Ikeda, T.; Kuroda, M.; Ohya, K.; Hara-Kudo, Y.; et al. Antimicrobial Resistance in Salmonella Isolated from Food Workers and Chicken Products in Japan. Antibiotics 2021, 10, 1541. https://doi.org/ 10.3390/antibiotics10121541

Academic Editor:

Alain Bousquet-Mélou

Received: 30 November 2021 Accepted: 14 December 2021 Published: 16 December 2021

Publisher's Note: MDPI stays neutral with regard to jurisdictional claims in published maps and institutional affiliations.

Copyright: (c) 2021 by the authors. Licensee MDPI, Basel, Switzerland. This article is an open access article distributed under the terms and conditions of the Creative Commons Attribution (CC BY) license (https:// creativecommons.org/licenses/by/ $4.0 /)$.

\begin{abstract}
Salmonella is an enteric bacterial pathogen that causes foodborne illness in humans. Thirdgeneration cephalosporin (TGC) resistance in Salmonella remains a global concern. Food workers may represent a reservoir of Salmonella, thus potentially contaminating food products. Therefore, we aimed to investigate the prevalence of Salmonella in food workers and characterize the isolates by serotyping and antimicrobial susceptibility testing. Salmonella was isolated from $583(0.079 \%)$ of 740,635 stool samples collected from food workers between January and December 2018, and then serotyped into 76 Salmonella enterica serovars and 22 untypeable Salmonella strains. High rates of antimicrobial resistance were observed for streptomycin (51.1\%), tetracycline (33.1\%), and kanamycin $(18.4 \%)$. Although isolates were susceptible to ciprofloxacin, 12 (2.1\%) strains (one $S$. Infantis, one $S$. Manhattan, two S. Bareilly, two S. Blockley, two S. Heidelberg, two S. Minnesota, one S. Goldcoast, and one untypeable Salmonella strain) were resistant to the TGC cefotaxime, all of which harbored $\beta$-lactamase genes ( $b l a_{\mathrm{CMY}-2}, b l a_{\mathrm{CTX}-\mathrm{M}-15}, b l a_{\mathrm{CTX}-\mathrm{M}-55}$, and $\left.b l a_{\mathrm{TEM}-52 \mathrm{~B}}\right)$. Moreover, $1.3 \%$ (4/309) of Salmonella strains (three $S$. Infantis and one $S$. Manhattan strains) isolated from chicken products were resistant to cefotaxime and harbored bla $_{\mathrm{CMY}-2}$ or $b l a_{\mathrm{TEM}-52 \mathrm{~B}}$. Thus, food workers may acquire TGCresistant Salmonella after the ingestion of contaminated chicken products and further contaminate food products.
\end{abstract}

Keywords: antimicrobial resistance; Salmonella; food worker; chicken product

\section{Introduction}

Non-typhoidal Salmonella is an enteric bacterial pathogen that causes foodborne illnesses worldwide. Salmonellosis is caused by non-typhoidal Salmonella enterica serovars such as Salmonella enterica subsp. enterica serovar Enteritidis, Salmonella Typhimurium, and Salmonella Infantis. Invasive non-typhoidal Salmonella is estimated to cause 3.4 million cases of infections annually, worldwide [1]. Although non-typhoidal Salmonella infections typically cause acute self-limiting gastroenteritis, they may cause invasive bacteremia, with severe symptoms in certain cases. Antimicrobial therapy is typically not provided in many cases of salmonellosis. However, antimicrobial therapy may be lifesaving in 
patients with bacteremia or an extra-intestinal focal infection. Ampicillin, third-generation cephalosporins (TGCs), fluoroquinolones, and trimethoprim-sulfamethoxazole are commonly used to treat salmonellosis [2]. Therefore, antimicrobial-resistant Salmonella poses an important issue in the chemotherapy treatment of humans.

A primary route of Salmonella transmission involves ingestion of contaminated chicken meat and eggs [3]. Mori et al. [4] isolated Salmonella from 286 (55.9\%) of 512 chicken meat samples collected in Japan between June 2015 and January 2016. In their study, the most frequently isolated serovar was $S$. Infantis, followed by $S$. Schwarzengrund, together accounting for $78.2 \%(243 / 311)$ of the isolates; $74.9 \%(182 / 243)$ of these two serovars are resistant to two or more antimicrobials, and seven $S$. Infantis strains were resistant to cefotaxime, a TGC. TGC-resistant Salmonella has additionally been isolated from fecal samples from healthy adults engaged in food handling work and diarrheic patients in Japan between 2012 and 2015 [5,6]. TGCs are classified as "critically important" by the World Health Organization [7]. In Japan, ceftiofur, a TGC, was approved in 1996 and was used for chemotherapy in cattle and pigs with bacterial infections. Moreover, the off-label use of ceftiofur in combination with in-ovo vaccination or vaccination of newly hatched chicks was performed in certain hatcheries until its use was abandoned in March 2012 [8]. The recent isolation of TGC-resistant Salmonella from chicken meat revealed TGC-resistant Salmonella may have survived in hatcheries and chicken farms after the withdrawal of ceftiofur in hatcheries. Food workers including food handlers who work in food and beverage companies that are infected with antimicrobial-resistant Salmonella after consuming or handling contaminated food may serve as reservoirs and pose a risk for food contamination. Therefore, in this study, we aimed to clarify the prevalence of Salmonella in food workers and characterize the isolates through serotyping and antimicrobial susceptibility testing. To estimate the origin of the isolates, we characterized Salmonella strains isolated from local chicken products by serotyping and antimicrobial susceptibility testing, and then compared the characteristics between human- and chicken-derived strains. Based on the findings of this study, the prevalence of Salmonella in stool samples of food workers was $0.079 \%$ between January and December 2018. Additionally, food workers may represent a reservoir of TGC-resistant Salmonella.

\section{Results}

\subsection{Salmonella Prevalence in Human Stools and Local Chicken Products}

Salmonella was isolated from $583(0.079 \%, 95 \%$ confidence interval $=0.072-0.085)$ of 740,635 human stool samples. The monthly prevalence of Salmonella ranged from $0.050 \%$ $(32 / 63,727)$ in January to $0.0125 \%(78 / 62,417)$ in September. The prevalence of Salmonella in human stool samples was significantly greater $(0.101 \%$; $188 / 185,718$; Fisher's exact test, $p<0.01)$ from August to October than from January to March $(0.067 \% ; 125 / 186,208)$. Salmonella-positive samples were obtained from 45 of 47 prefectures in Japan. The strains isolated from humans were serotyped into 76 serovars and 22 untypeable Salmonella isolates (Table 1). The top 14 serovars (S. Schwarzengrund to $S$. Typhimurium) together accounted for over $70 \%$ of human-derived strains. Of 235 local chicken products, Salmonella was isolated from $200(85.1 \%, 95 \%$ confidence interval $=79.9-89.4)$. To estimate the origin of the isolates, we characterized Salmonella strains isolated from local chicken products. Chicken-derived strains were serotyped into five serovars and two untypeable Salmonella strains. The top three serovars were S. Schwarzengrund (73.0\%), S. Infantis (15.0\%), and S. Manhattan (8.5\%), together accounting for $96.5 \%$ of the chicken-derived strains. 
Table 1. Number and serovars of Salmonella strains isolated from humans and chicken products.

\begin{tabular}{|c|c|c|c|c|}
\hline \multirow{2}{*}{ Serovar } & \multicolumn{2}{|c|}{ Human } & \multicolumn{2}{|c|}{ Chicken } \\
\hline & $\mathbf{N}$ & $\%$ & $\mathbf{N}$ & $\%$ \\
\hline Schwarzengrund & 99 & 17.0 & 146 & 73.0 \\
\hline Infantis & 50 & 8.6 & 30 & 15.0 \\
\hline Typhimurium monophasic variant & 37 & 6.3 & 0 & 0.0 \\
\hline Thompson & 36 & 6.2 & 0 & 0.0 \\
\hline Cubana & 26 & 4.5 & 0 & 0.0 \\
\hline Manhattan & 25 & 4.3 & 17 & 8.5 \\
\hline Newport & 25 & 4.3 & 0 & 0.0 \\
\hline Mbandaka & 20 & 3.4 & 0 & 0.0 \\
\hline Agona & 17 & 2.9 & 4 & 2.0 \\
\hline Bareilly & 16 & 2.7 & 0 & 0.0 \\
\hline Braenderup & 16 & 2.7 & 0 & 0.0 \\
\hline Corvallis & 16 & 2.7 & 0 & 0.0 \\
\hline Enteritidis & 14 & 2.4 & 0 & 0.0 \\
\hline Typhimurium & 13 & 2.2 & 0 & 0.0 \\
\hline Saintpaul & 9 & 1.5 & 0 & 0.0 \\
\hline Anatum & 8 & 1.4 & 1 & 0.5 \\
\hline Stanley & 7 & 1.2 & 0 & 0.0 \\
\hline Litchfield & 6 & 1.0 & 0 & 0.0 \\
\hline Rissen & 6 & 1.0 & 0 & 0.0 \\
\hline Senftenberg & 6 & 1.0 & 0 & 0.0 \\
\hline Blockley & 5 & 0.9 & 0 & 0.0 \\
\hline Weltevreden & 5 & 0.9 & 0 & 0.0 \\
\hline Heidelberg & 5 & 0.9 & 0 & 0.0 \\
\hline Derby & 4 & 0.7 & 0 & 0.0 \\
\hline Hadar & 4 & 0.7 & 0 & 0.0 \\
\hline Panama & 4 & 0.7 & 0 & 0.0 \\
\hline Paratyphi B & 4 & 0.7 & 0 & 0.0 \\
\hline Colindale & 3 & 0.5 & 0 & 0.0 \\
\hline London & 3 & 0.5 & 0 & 0.0 \\
\hline Montevideo & 3 & 0.5 & 0 & 0.0 \\
\hline Muenchen & 3 & 0.5 & 0 & 0.0 \\
\hline Oranienburg & 3 & 0.5 & 0 & 0.0 \\
\hline Othmarschen & 3 & 0.5 & 0 & 0.0 \\
\hline Potsdam & 3 & 0.5 & 0 & 0.0 \\
\hline Uganda & 3 & 0.5 & 0 & 0.0 \\
\hline Altona & 2 & 0.3 & 0 & 0.0 \\
\hline Brandenburg & 2 & 0.3 & 0 & 0.0 \\
\hline Bredeney & 2 & 0.3 & 0 & 0.0 \\
\hline Cerro & 2 & 0.3 & 0 & 0.0 \\
\hline Duesseldorf & 2 & 0.3 & 0 & 0.0 \\
\hline Havana & 2 & 0.3 & 0 & 0.0 \\
\hline Lexington & 2 & 0.3 & 0 & 0.0 \\
\hline Liverpool & 2 & 0.3 & 0 & 0.0 \\
\hline Minnesota & 2 & 0.3 & 0 & 0.0 \\
\hline Narashino & 2 & 0.3 & 0 & 0.0 \\
\hline Poona & 2 & 0.3 & 0 & 0.0 \\
\hline Singapore & 2 & 0.3 & 0 & 0.0 \\
\hline Virchow & 2 & 0.3 & 0 & 0.0 \\
\hline Others ( 28 serovars and 22 untypeable) & 50 & 8.6 & 2 & 1.0 \\
\hline Total & 583 & & 200 & \\
\hline
\end{tabular}

\subsection{Antimicrobial Susceptibility}

Antimicrobial susceptibility testing of human-derived strains showed that $333(57.1 \%)$ of the 583 strains were resistant to at least one antimicrobial, and $204(35.0 \%)$ isolates were resistant to two or more antimicrobials. High rates of antimicrobial resistance were observed for streptomycin (51.1\%), tetracycline (33.1\%), and kanamycin (18.4\%) (Table 2). 
All isolates were susceptible to ciprofloxacin. In total, $(2.1 \%, 12 / 583) 12$ strains (one $S$. Infantis, one $S$. Manhattan, two $S$. Bareilly, two $S$. Blockley, two $S$. Heidelberg, two $S$. Minnesota, one $S$. Goldcoast, and one untypeable Salmonella strain) were resistant to cefotaxime. The cefotaxime-resistant untypeable strain agglutinated with anti-H:r, anti-H:1, and anti-H:5 sera, but not with anti-O sera. Multidrug-resistant strains isolated from human stool samples such as $S$. Schwarzengrund, $S$. Infantis, $S$. Typhimurium monophasic variant, and S. Manhattan accounted for $86.9 \%, 28.0 \%, 75.7 \%$, and $88.0 \%$, respectively, together accounting for $73.5 \%(150 / 204)$ (Table 3). Among S. Schwarzengrund strains, the prevalence of strains resistant to streptomycin, kanamycin, and tetracycline was greatest $(58.6 \%)$, followed by streptomycin and tetracycline $(11.1 \%)$. Among S. Infantis strains, the prevalence of strains susceptible to antimicrobials tested was greatest $(40.0 \%)$, followed by streptomycin (32.0\%). Among S. Typhimurium monophasic variants, the prevalence of strains resistant to ampicillin, streptomycin, and tetracycline was greatest (45.9\%). Among $S$. Manhattan strains, the prevalence of strains resistant to streptomycin and tetracycline was greatest $(60.0 \%)$. In contrast, multidrug-resistant strains were not observed among $S$. Thompson, $S$. Cubana, and $S$. Newport strains.

Antimicrobial susceptibility testing of chicken-derived strains showed that $174(87.0 \%)$ of the 200 strains were resistant to at least one antimicrobial, and 141 (70.5\%) strains were resistant to two or more antimicrobials. High antimicrobial resistance rates were observed for streptomycin $(73.0 \%)$, tetracycline $(67.5 \%)$, and kanamycin $(53.5 \%)$. Four $(1.3 \%)$ strains (three $S$. Infantis and one $S$. Manhattan strain) were resistant to cefotaxime. The three products contaminated with these cefotaxime-resistant $S$. Infantis strains were transported from the same chicken-processing plant on different days. The product contaminated with cefotaxime-resistant $S$. Manhattan was transferred from a different chicken processing plant. Among S. Schwarzengrund strains, the prevalence of strains resistant to streptomycin, kanamycin, and tetracycline was greatest (32.2\%), sharing similarity with human-derived strains. Among $S$. Manhattan strains, the prevalence of strains resistant to streptomycin and tetracycline was greatest $(50.0 \%)$, bearing similarity with human-derived strains. Among $S$. Infantis strains, the prevalence of strains resistant to streptomycin and tetracycline was greatest $(36.7 \%)$, which differed from that in human-derived strains. All strains were susceptible to colistin and ciprofloxacin.

Whole-genome sequencing (WGS) analysis revealed that all 16 cefotaxime-resistant strains isolated from human stool samples and chicken products harbored $\beta$-lactamase (bla) genes (Table 4). Of the 16 strains, 8 (4 S. Infantis, 2 S. Minnesota, 1 S. Heidelberg, and 1 untypeable Salmonella strain) harbored $b l a_{\mathrm{CMY}-2}$. Two $S$. Blockley strains harbored $b l a_{\mathrm{CTX}-\mathrm{M}-15}$. One $S$. Heidelberg strain harbored $b l a_{\mathrm{LAT}-3}$. Two $S$. Bareilly strains harbored $b l a_{\mathrm{CTX}-\mathrm{M}-55}$. Two $S$. Manhattan strains harbored $b l a_{\mathrm{TEM}-52 \mathrm{~B}}$, and one $S$. Goldcoast strain harbored $b l a_{\mathrm{CTX}-\mathrm{M}-55}, b l a_{\mathrm{LAP}-2,}$ and $b l a_{\mathrm{TEM}-1}$. 
Table 2. Antimicrobial susceptibility of Salmonella isolates from humans and chicken products.

\begin{tabular}{|c|c|c|c|c|c|c|c|c|c|c|c|c|c|c|c|c|c|c|c|c|c|c|}
\hline \multirow{2}{*}{ Serovar } & \multirow{2}{*}{ Origin } & \multirow{2}{*}{$\mathbf{N}$} & \multicolumn{2}{|c|}{ ABPC } & \multicolumn{2}{|c|}{ CEZ } & \multicolumn{2}{|c|}{ CTX } & \multicolumn{2}{|c|}{ SM } & \multicolumn{2}{|c|}{ GM } & \multicolumn{2}{|c|}{$\mathbf{K M}$} & \multicolumn{2}{|c|}{ TC } & \multicolumn{2}{|c|}{ NA } & \multicolumn{2}{|c|}{$\overline{C L}$} & \multicolumn{2}{|c|}{$\mathrm{CP}$} \\
\hline & & & $\mathbf{N}$ & $\%$ & $\mathbf{N}$ & $\%$ & $\mathbf{N}$ & $\%$ & $\mathbf{N}$ & $\%$ & $\mathbf{N}$ & $\%$ & $\mathbf{N}$ & $\%$ & $\mathbf{N}$ & $\%$ & $\mathbf{N}$ & $\%$ & $\mathbf{N}$ & $\%$ & $\mathbf{N}$ & $\%$ \\
\hline \multirow{2}{*}{ Schwarzengrund } & Human & 99 & 0 & 0.0 & 0 & 0.0 & 0 & 0.0 & 85 & 85.9 & 0 & 0.0 & 81 & 81.8 & 83 & 83.8 & 16 & 16.2 & 0 & 0.0 & 0 & 0.0 \\
\hline & Chicken & 146 & 1 & 0.7 & 0 & 0.0 & 0 & 0.0 & 99 & 67.8 & 0 & 0.0 & 93 & 63.7 & 93 & 63.7 & 24 & 16.4 & 0 & 0.0 & 2 & 1.4 \\
\hline \multirow[t]{2}{*}{ Infantis } & Human & 50 & 1 & 2.0 & 1 & 2.0 & 1 & 2.0 & 30 & 60.0 & 0 & 0.0 & 8 & 16.0 & 13 & 26.0 & 1 & 2.0 & 0 & 0.0 & 0 & 0.0 \\
\hline & Chiciken & 30 & 4 & 13.3 & 3 & 10.0 & 3 & 10.0 & 26 & 86.7 & 1 & 3.3 & 10 & 33.3 & 24 & 80.0 & 4 & 13.3 & 0 & 0.0 & 0 & 0.0 \\
\hline $\begin{array}{l}\text { Typhimurium } \\
\text { monophasic variant }\end{array}$ & Human & 37 & 21 & 56.8 & 1 & 2.7 & 0 & 0.0 & 29 & 78.4 & 1 & 2.7 & 1 & 2.7 & 29 & 78.4 & 1 & 2.7 & 0 & 0.0 & 2 & 5.4 \\
\hline Thompson & Human & 36 & 0 & 0.0 & 0 & 0.0 & 0 & 0.0 & 21 & 58.3 & 0 & 0.0 & 0 & 0.0 & 0 & 0.0 & 0 & 0.0 & 0 & 0.0 & 0 & 0.0 \\
\hline \multirow[t]{2}{*}{ Manhattan } & Human & 25 & 5 & 20.0 & 1 & 4.0 & 1 & 4.0 & 24 & 96.0 & 0 & 0.0 & 0 & 0.0 & 22 & 88.0 & 6 & 24.0 & 0 & 0.0 & 0 & 0.0 \\
\hline & Chicken & 17 & 1 & 5.9 & 1 & 5.9 & 1 & 5.9 & 15 & 88.2 & 0 & 0.0 & 1 & 5.9 & 12 & 70.6 & 1 & 5.9 & 0 & 0.0 & 0 & 0.0 \\
\hline Newport & Human & 25 & 0 & 0.0 & 0 & 0.0 & 0 & 0.0 & 3 & 12.0 & 0 & 0.0 & 0 & 0.0 & 0 & 0.0 & 0 & 0.0 & 0 & 0.0 & 0 & 0.0 \\
\hline Mbandaka & Human & 20 & 0 & 0.0 & 0 & 0.0 & 0 & 0.0 & 8 & 40.0 & 0 & 0.0 & 0 & 0.0 & 1 & 5.0 & 0 & 0.0 & 0 & 0.0 & 0 & 0.0 \\
\hline \multirow[t]{2}{*}{ Agona } & Human & 17 & 1 & 5.9 & 1 & 5.9 & 0 & 0.0 & 9 & 52.9 & 0 & 0.0 & 0 & 0.0 & 10 & 58.8 & 2 & 11.8 & 0 & 0.0 & 1 & 5.9 \\
\hline & Chicken & 4 & 0 & 0.0 & 0 & 0.0 & 0 & 0.0 & 4 & 100.0 & 0 & 0.0 & 1 & 25.0 & 4 & 100.0 & 0 & 0.0 & 0 & 0.0 & 1 & 25.0 \\
\hline Bareilly & Human & 16 & 2 & 12.5 & 2 & 12.5 & 2 & 12.5 & 5 & 31.3 & 2 & 12.5 & 0 & 0.0 & 0 & 0.0 & 0 & 0.0 & 0 & 0.0 & 0 & 0.0 \\
\hline Braenderup & Human & 16 & 0 & 0.0 & 0 & 0.0 & 0 & 0.0 & 12 & 75.0 & 0 & 0.0 & 0 & 0.0 & 0 & 0.0 & 2 & 12.5 & 0 & 0.0 & 0 & 0.0 \\
\hline Corvallis & Human & 16 & 0 & 0.0 & 0 & 0.0 & 0 & 0.0 & 1 & 6.3 & 0 & 0.0 & 0 & 0.0 & 0 & 0.0 & 0 & 0.0 & 0 & 0.0 & 0 & 0.0 \\
\hline Typhimurium & Human & 13 & 0 & 0.0 & 0 & 0.0 & 0 & 0.0 & 2 & 15.4 & 0 & 0.0 & 0 & 0.0 & 1 & 7.7 & 0 & 0.0 & 0 & 0.0 & 0 & 0.0 \\
\hline \multirow[t]{2}{*}{ Others } & Human & 173 & 20 & 11.6 & 9 & 5.2 & 8 & 4.6 & 67 & 38.7 & 2 & 1.2 & 17 & 9.8 & 34 & 19.7 & 11 & 6.4 & 0 & 0.0 & 10 & 5.8 \\
\hline & Chicken & 3 & 0 & 0.0 & 0 & 0.0 & 0 & 0.0 & 2 & 66.7 & 0 & 0.0 & 2 & 66.7 & 2 & 66.7 & 0 & 0.0 & 0 & 0.0 & 0 & 0.0 \\
\hline \multirow[t]{2}{*}{ Total } & Human & 583 & 51 & 8.7 & 15 & 2.6 & 12 & 2.1 & 298 & 51.1 & 5 & 0.9 & 107 & 18.4 & 193 & 33.1 & 41 & 7.0 & 2 & 0.3 & 13 & 2.2 \\
\hline & Chicken & 200 & 6 & 3.0 & 4 & 2.0 & 4 & 2.0 & 146 & 73.0 & 1 & 0.5 & 107 & 53.5 & 135 & 67.5 & 29 & 14.5 & 0 & 0.0 & 3 & 1.5 \\
\hline
\end{tabular}

ABPC: ampicillin, CEZ: cefazolin, CTX: cefotaxime, SM: streptomycin, GM: gentamycin, KM: kanamycin, TC: tetracycline, NA: nalidixic acid, CL: colistin, CP: chloramphenicol. 
Table 3. Antimicrobial resistance profiles of the six most-frequent Salmonella enterica serovars isolated from human stools and chicken products.

\begin{tabular}{|c|c|c|c|c|c|}
\hline \multirow{2}{*}{ Serovar } & \multirow{2}{*}{ Antimicrobial Resistance Profile } & \multicolumn{2}{|c|}{ Human } & \multicolumn{2}{|c|}{ Chicken } \\
\hline & & No. & $\%$ & No. & $\%$ \\
\hline \multirow[t]{15}{*}{ Schwarzengr } & & 99 & & 146 & \\
\hline & $\mathrm{ABPC}+\mathrm{SM}+\mathrm{KM}+\mathrm{TC}+\mathrm{NA}+\mathrm{CP}$ & 0 & 0.0 & 1 & 0.7 \\
\hline & $\mathrm{SM}+\mathrm{KM}+\mathrm{TC}+\mathrm{NA}+\mathrm{CP}$ & 0 & 0.0 & 1 & 0.7 \\
\hline & $\mathrm{SM}+\mathrm{KM}+\mathrm{TC}+\mathrm{NA}$ & 8 & 8.1 & 16 & 11.0 \\
\hline & $\mathrm{KM}+\mathrm{TC}+\mathrm{NA}$ & 1 & 1.0 & 0 & 0.0 \\
\hline & $\mathrm{SM}+\mathrm{KM}+\mathrm{NA}$ & 1 & 1.0 & 1 & 0.7 \\
\hline & $\mathrm{SM}+\mathrm{TC}+\mathrm{NA}$ & 5 & 5.1 & 3 & 2.1 \\
\hline & $\mathrm{SM}+\mathrm{KM}+\mathrm{TC}$ & 58 & 58.6 & 47 & 32.2 \\
\hline & $\mathrm{SM}+\mathrm{TC}$ & 11 & 11.1 & 24 & 16.4 \\
\hline & $\mathrm{SM}+\mathrm{KM}$ & 1 & 1.0 & 3 & 2.1 \\
\hline & $\mathrm{KM}+\mathrm{NA}$ & 1 & 1.0 & 2 & 1.4 \\
\hline & $\mathrm{KM}+\mathrm{TC}$ & 0 & 0.0 & 1 & 0.7 \\
\hline & KM & 11 & 11.1 & 21 & 14.4 \\
\hline & SM & 1 & 1.0 & 3 & 2.1 \\
\hline & susceptible & 1 & 1.0 & 23 & 15.8 \\
\hline \multirow[t]{13}{*}{ Infantis } & & 50 & & 30 & \\
\hline & $\mathrm{ABPC}+\mathrm{SM}+\mathrm{GM}+\mathrm{KM}+\mathrm{TC}+\mathrm{NA}$ & 0 & 0.0 & 1 & 3.3 \\
\hline & $\mathrm{ABPC}+\mathrm{CEZ}+\mathrm{CTX}+\mathrm{SM}+\mathrm{KM}+\mathrm{TC}$ & 1 & 2.0 & 0 & 0.0 \\
\hline & $\mathrm{ABPC}+\mathrm{CEZ}+\mathrm{CTX}+\mathrm{SM}+\mathrm{TC}$ & 0 & 0.0 & 3 & 10.0 \\
\hline & $\mathrm{SM}+\mathrm{KM}+\mathrm{TC}+\mathrm{NA}$ & 0 & 0.0 & 1 & 3.3 \\
\hline & $\mathrm{SM}+\mathrm{KM}+\mathrm{TC}$ & 6 & 12.0 & 6 & 20.0 \\
\hline & $\mathrm{SM}+\mathrm{TC}+\mathrm{NA}$ & 1 & 2.0 & 2 & 6.7 \\
\hline & $\mathrm{SM}+\mathrm{TC}$ & 5 & 10.0 & 11 & 36.7 \\
\hline & $\mathrm{SM}+\mathrm{KM}$ & 1 & 2.0 & 0 & 0.0 \\
\hline & TC & 0 & 0.0 & 0 & 0.0 \\
\hline & KM & 0 & 0.0 & 2 & 6.7 \\
\hline & SM & 16 & 32.0 & 2 & 6.7 \\
\hline & susceptible & 20 & 40.0 & 2 & 6.7 \\
\hline \multicolumn{2}{|c|}{ Typhimurium monophasic variant } & 37 & & 0 & \\
\hline & $\mathrm{SM}+\mathrm{GM}+\mathrm{KM}+\mathrm{TC}$ & 1 & 2.7 & 0 & 0.0 \\
\hline & $\mathrm{ABPC}+\mathrm{CEZ}+\mathrm{SM}+\mathrm{TC}$ & 1 & 2.7 & 0 & 0.0 \\
\hline & $\mathrm{ABPC}+\mathrm{SM}+\mathrm{TC}+\mathrm{CP}$ & 1 & 2.7 & 0 & 0.0 \\
\hline & $\mathrm{ABPC}+\mathrm{SM}+\mathrm{NA}$ & 1 & 2.7 & 0 & 0.0 \\
\hline & $\mathrm{SM}+\mathrm{TC}+\mathrm{CP}$ & 1 & 2.7 & 0 & 0.0 \\
\hline & $\mathrm{ABPC}+\mathrm{SM}+\mathrm{TC}$ & 17 & 45.9 & 0 & 0.0 \\
\hline & $\mathrm{ABPC}+\mathrm{SM}$ & 1 & 2.7 & 0 & 0.0 \\
\hline & $\mathrm{SM}+\mathrm{TC}$ & 5 & 13.5 & 0 & 0.0 \\
\hline & $\mathrm{TC}$ & 3 & 8.1 & 0 & 0.0 \\
\hline & $\mathrm{SM}$ & 1 & 2.7 & 0 & 0.0 \\
\hline & susceptible & 5 & 13.5 & 0 & 0.0 \\
\hline \multirow[t]{3}{*}{ Thompson } & & 36 & & 0 & \\
\hline & $\mathrm{SM}$ & 21 & 58.3 & 0 & 0.0 \\
\hline & susceptible & 15 & 41.7 & 0 & 0.0 \\
\hline \multirow[t]{3}{*}{ Cubana } & & 26 & & 0 & \\
\hline & SM & 1 & 3.8 & 0 & 0.0 \\
\hline & susceptible & 25 & 96.2 & 0 & 0.0 \\
\hline \multirow[t]{10}{*}{ Manhattan } & & 25 & & 17 & \\
\hline & $\mathrm{ABPC}+\mathrm{CEZ}+\mathrm{CTX}+\mathrm{SM}+\mathrm{TC}$ & 1 & 4.0 & 1 & 5.0 \\
\hline & $\mathrm{ABPC}+\mathrm{SM}+\mathrm{TC}+\mathrm{NA}$ & 4 & 16.0 & 0 & 0.0 \\
\hline & $\mathrm{SM}+\mathrm{TC}+\mathrm{NA}$ & 2 & 8.0 & 0 & 0.0 \\
\hline & $\mathrm{SM}+\mathrm{NA}$ & 0 & 0.0 & 1 & 5.0 \\
\hline & $\mathrm{SM}+\mathrm{TC}$ & 15 & 60.0 & 10 & 50.0 \\
\hline & $\mathrm{TC}$ & 0 & 0.0 & 1 & 5.0 \\
\hline & KM & 0 & 0.0 & 1 & 5.0 \\
\hline & SM & 2 & 8.0 & 3 & 15.0 \\
\hline & susceptible & 1 & 4.0 & 0 & 0.0 \\
\hline
\end{tabular}


Table 4. Antimicrobial resistant genes in cefotaxime-resistant strains.

\begin{tabular}{|c|c|c|c|c|c|c|}
\hline Serovar & Source & Strain & ST & $\begin{array}{c}\text { Antimicrobial } \\
\text { Resistance Profile }\end{array}$ & bla Gene & $\begin{array}{l}\text { Other Antimicrobial } \\
\text { Resistant Genes }\end{array}$ \\
\hline \multicolumn{7}{|l|}{ Infantis } \\
\hline & Human & BM-114 & 32 & $\begin{array}{c}\text { ABPC, CEZ, CTX, SM, KM, } \\
\text { TC }\end{array}$ & CMY-2 & $\begin{array}{c}a a c\left(6^{\prime}\right)-I a a, \operatorname{ant}\left(3^{\prime \prime}\right)-I a, \operatorname{aph}\left(3^{\prime}\right)-I a \\
q a c E d e l t a 1, \operatorname{sul} 1, \operatorname{tet}(\mathrm{A})\end{array}$ \\
\hline & Chicken & B-21 & 32 & ABPC, CEZ, CTX, SM, TC & CMY-2 & $\begin{array}{c}\text { aac }\left(6^{\prime}\right)-\text { Iaa }, \operatorname{ant}\left(3^{\prime \prime}\right)-\text { Ia }, d f r A 14 \\
\text { qacEdelta1, sul1, tet }(\mathrm{A})\end{array}$ \\
\hline & Chicken & M-4 & 32 & ABPC, CEZ, CTX, SM, TC & CMY-2 & $\begin{array}{c}\text { aac }\left(6^{\prime}\right) \text {-Iaa, ant }\left(3^{\prime \prime}\right) \text {-Ia, qacEdelta1, } \\
\text { sul1, tet }(\mathrm{A})\end{array}$ \\
\hline & Chicken & M-5 & 32 & ABPC, CEZ, CTX, SM, TC & CMY-2 & $\begin{array}{c}\text { aac }\left(6^{\prime}\right)-\text { Iaa, ant }\left(3^{\prime \prime}\right)-\operatorname{Ia}, \operatorname{dfr} A 14 \\
\text { qacEdelta1, sul1, tet }(\mathrm{A})\end{array}$ \\
\hline \multicolumn{7}{|r|}{ 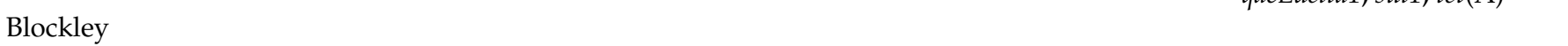 } \\
\hline & Human & TK-117 & 52 & $\begin{array}{c}\mathrm{ABPC}, \mathrm{CEZ}, \mathrm{CTX}, \mathrm{SM}, \mathrm{KM} \text {, } \\
\text { TC, CP }\end{array}$ & CTX-M-15 & $\begin{array}{l}\operatorname{aac}\left(6^{\prime}\right)-I a a, \operatorname{aph}\left(3^{\prime \prime}\right)-I b, a p h\left(3^{\prime}\right)-I a, \\
\operatorname{aph}(6)-I d, \operatorname{cat} A 2, \operatorname{mph}(\mathrm{A}), \operatorname{tet}(\mathrm{A})\end{array}$ \\
\hline & Human & TK-120 & 52 & $\begin{array}{c}\text { ABPC, CEZ, CTX, SM, KM, } \\
\text { TC, CP }\end{array}$ & CTX-M-15 & $\begin{array}{l}\operatorname{aac}\left(6^{\prime}\right)-I a a, \operatorname{aph}\left(3^{\prime \prime}\right)-I b, a p h\left(3^{\prime}\right)-I a, \\
\operatorname{aph}(6)-I d, \operatorname{cat} A 2, m p h(\mathrm{~A}), \operatorname{tet}(\mathrm{A})\end{array}$ \\
\hline \multicolumn{7}{|l|}{ Minnesota } \\
\hline & Human & TK-227 & 52 & ABPC, CEZ, CTX, KM, TC & CMY-2 & $\operatorname{aac}\left(6^{\prime}\right)-I a a, \operatorname{aph}\left(3^{\prime}\right)-I a, \operatorname{sul} 2$, tet $(\mathrm{A})$ \\
\hline & Human & TK-256 & 548 & $\begin{array}{c}\text { ABPC, CEZ, CTX, KM, TC, } \\
\text { NA }\end{array}$ & CMY-2 & $\operatorname{aac}\left(6^{\prime}\right)-I a a, q n r B 19, \operatorname{sul} 2$, tet $(\mathrm{A})$ \\
\hline \multicolumn{7}{|l|}{ Heidelberg } \\
\hline & Human & TK-124 & 15 & $\begin{array}{c}\text { ABPC, CEZ, CTX, SM, GM, } \\
\text { TC, NA }\end{array}$ & LAT-3 & $\begin{array}{c}a a c(3)-V I a, a a c\left(6^{\prime}\right)-I a a, \text { ant }\left(3^{\prime \prime}\right)-I a, \\
\text { fosA7, qacEdelta1, sul1, sul2, } \\
\text { tet(A) }\end{array}$ \\
\hline & Human & TK-167 & 15 & $\mathrm{ABPC}, \mathrm{CEZ}, \mathrm{CTX}, \mathrm{TC}, \mathrm{NA}$ & CMY-2 & $\operatorname{aac}\left(6^{\prime}\right)-I a a$, fos $A 7$, sul2, tet(A) \\
\hline Bareııy & Human & BM-153 & 203 & $\begin{array}{c}\text { ABPC, CEZ, CTX, SM, GM, } \\
\text { TMP }\end{array}$ & CTX-M-55 & $\begin{array}{c}a a c(3)-I I d, a a c\left(6^{\prime}\right)-\operatorname{Iaa}, \operatorname{ant}\left(3^{\prime \prime}\right)-I a \\
\operatorname{dfr} A 14, \text { fos } A 4, \operatorname{lnu}(\mathrm{F}), \operatorname{mph}(\mathrm{A}), \\
q n r S 13\end{array}$ \\
\hline & Human & BM-226 & 203 & $\begin{array}{c}\text { ABPC, CEZ, CTX, SM, GM, } \\
\text { TMP }\end{array}$ & CTX-M-55 & $\begin{array}{c}\operatorname{aac}(3)-I I d, \operatorname{aac}\left(6^{\prime}\right)-\operatorname{Iaa}, \operatorname{ant}\left(3^{\prime \prime}\right)-I a \\
\operatorname{dfr} A 14, \text { fos } A 4, \operatorname{lnu}(\mathrm{F}), \operatorname{mph}(\mathrm{A}), \\
q n r S 13\end{array}$ \\
\hline \multicolumn{7}{|l|}{ Manhattan } \\
\hline & Human & BM-136 & 18 & ABPC, CEZ, CTX, SM, TC & TEM-52B & $\begin{array}{c}\text { aac }\left(6^{\prime}\right) \text {-Iaa, ant }\left(3^{\prime \prime}\right) \text {-Ia, qacEdelta1, } \\
\text { sul1, tet }(\mathrm{A})\end{array}$ \\
\hline & Chicken & $\mathrm{K}-1$ & 18 & ABPC, CEZ, CTX, SM, TC & TEM-52B & $\begin{array}{c}\text { aac }\left(6^{\prime}\right) \text {-Iaa, ant }\left(3^{\prime \prime}\right) \text {-Ia, qacEdelta1, } \\
\text { sul1, tet }(\mathrm{A})\end{array}$ \\
\hline \multicolumn{7}{|l|}{ Goldcoast } \\
\hline & Human & BM-274 & 358 & $\begin{array}{l}\text { ABPC, CEZ, CTX, GM, } \\
\text { KM, TC, NA, CP }\end{array}$ & $\begin{array}{l}\text { CTX-M-55, } \\
\text { LAP-2, TEM-1 }\end{array}$ & $\begin{array}{c}\text { ARR-3, aac(3)-IId, aac (6')-Iaa, } \\
\text { aph(3')-Ia, aph(6)-Id, dfrA14, floR, } \\
q n r S 13, \text { sul2, sul3, tet(A) }\end{array}$ \\
\hline \multicolumn{7}{|l|}{ Untypable } \\
\hline & Human & TK-272 & 32 & ABPC, CEZ, CTX, SM, TC & CMY-2 & $\begin{array}{c}\text { aac }\left(6^{\prime}\right)-I a a, \text { ant }\left(3^{\prime \prime}\right)-I a, \text { qacEdelta1, } \\
\text { sul1, tet }(\mathrm{A})\end{array}$ \\
\hline
\end{tabular}

ST: sequence type.

\section{Discussion}

The results of the present study showed that the prevalence of Salmonella in stool samples of food workers was $0.079 \%$ between January and December 2018, and the prevalence was greater from August to October than that from January to March. Outbreaks of Salmonella-associated food poisoning in Japan occur year-round, peaking from May to November [3]. Xu et al. [9] have suggested Salmonella may cause human infections in symptomatic and asymptomatic states, as Salmonella strains isolated from symptomatic and asymptomatic individuals are genetically and phenotypically indistinguishable. Kariuki et al. [10] reported an elevated level of relatedness between Salmonella strains isolated from symptomatic patients and asymptomatic carriers by phylogenetic analysis. Food workers evaluated in the present study may have been infected with Salmonella via ingestion of foods that can cause food poisoning, although they were asymptomatic when submitting 
their stool samples. Excluding S. Typhimurium monophasic variants, S. Cubana, and $S$. Newport, the top 14 serovars in human-derived strains, are known to be prevalent in Japanese broiler and laying hen flocks [11,12]. Notably, three serovars, $S$. Schwarzengrund, $S$. Infantis, and S. Manhattan, accounted for over $98 \%$ of chicken-derived strains in the present study. The most common antimicrobial resistance profiles of $S$. Schwarzengrund and S. Manhattan in human-derived strains were the same as those in chicken-derived strains. However, the primary antimicrobial resistance profiles of $S$. Infantis in human- and chicken-derived strains were different. $S$. Infantis strains susceptible to all antimicrobials tested represented the most common isolates among human-derived strains, whereas they represented the second-most common strains among chicken-derived strains. In our previous study [11], S. Infantis represented the second-most common serovar among Salmonella strains isolated from Japanese laying hen flocks, with $92.9 \%$ of $S$. Infantis strains susceptible to all antimicrobials tested. Therefore, the distribution of antimicrobial resistance profiles in S. Infantis isolated from human stool samples in the present study may be attributed to strains originating from Japanese broilers and laying hen flocks. Shimojima et al. [13] recently reported that Salmonella prevalence was greater in local chicken products (57.9\%) than in imported products (8.5\%). According to the Ministry of Agriculture, Forestry and Fisheries of Japan [14], local chicken and chicken egg products accounted for approximately $65 \%$ of total chicken meat distribution and $96 \%$ of total chicken egg distribution, respectively, in Japan in FY 2018. The results of this study suggest many food workers may be infected with Salmonella via ingestion of local chicken and egg products. Moreover, as $S$. Typhimurium, its monophasic variant, and $S$. Newport are prevalent in local cattle and pigs [15], beef and pork may represent sources of Salmonella infections among food workers.

Of the 583 human-derived Salmonella strains isolated in the present study, $12(2.1 \%)$ were resistant to cefotaxime. Cefotaxime resistance was observed in seven serovars and one untypeable Salmonella strain. As the untypeable strain was assigned to sequence type 32, to which most $S$. Infantis strains belong, the strain may be a variant of $S$. Infantis. This assumption is based on multi-locus sequence typing (MLST) in accordance with public databases for molecular typing and microbial genome diversity (https: / / pubmlst.org, accessed on 23 November 2021). In the present study, cefotaximeresistant $S$. Infantis and $S$. Manhattan strains were obtained from local chicken products, and harbored the same bla genes $\left(b l_{\mathrm{CMY}-2}\right.$ or $\left.b l a_{\mathrm{TEM}-52 \mathrm{~B}}\right)$ as strains isolated from human stool samples. Shigemura et al. [16] reported that bla ${ }_{\mathrm{CMY}-2}$-harboring $S$. Infantis and

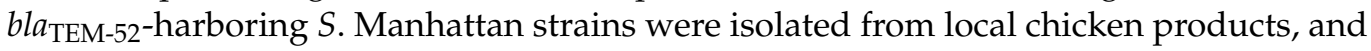
one $b l a_{\mathrm{CMY}-2}$-harboring $S$. Heidelberg strain was isolated from an imported chicken product. According to the Trade Statistics of Japan (https:/ /www.customs.go.jp/toukei/info/ index_e.htm, accessed on 23 November 2021), chicken products imported from Brazil accounted for approximately $75 \%$ of imported chicken products in 2018. In Brazil, S. Minnesota, $S$. Infantis, and $S$. Heidelberg are prevalent in broiler flocks and chicken products, some of which show TGC resistance [17-21]. Shimojima et al. [13] reported the cefotaxime resistance rate in Salmonella isolated between 2009 and 2017 was lower in local chicken products $(1.9 \%)$ than in imported chicken products (28.0\%); additionally, seven $S$. Heidelberg and three $S$. Minnesota strains, isolated from imported chicken products, contain bla genes. Moreover, in their study, bla-harboring Salmonella strains were not isolated from beef and pork samples. Therefore, local and imported chicken products may represent major sources of TGC-resistant Salmonella that infect food workers.

Shigemura et al. [22] recently reported the prevalence of Salmonella in human stool samples obtained from food workers between January and October 2017 was $0.113 \%$ $(164 / 145,220)$, and seven strains were resistant to TGCs. Of the seven TGC-resistant strains, one Salmonella Senftenberg and three Salmonella Haardt strains harbored bla $a_{\mathrm{CTX}-\mathrm{M}-14}$ and bla $_{\text {CTX-M-15, }}$ respectively. The remaining three strains, namely Salmonella Agona, S. Infantis, and an untypeable Salmonella strain, harbored bla $a_{\mathrm{CMY}-2}$. Although their detection method differed from ours, their results share similarity to those reported in the present study, suggesting food workers may be infected with TGC-resistant Salmonella after the ingestion 
of contaminated chicken products and subsequently serve as reservoirs. Although the prevalence of TGC-resistant Salmonella in food workers may be low, they routinely serve food to consumers. Moreover, estimating the prevalence and characteristics of Salmonella in the Japanese population including food workers is possible. Therefore, monitoring the prevalence and characteristics of Salmonella in food workers is useful for risk management of Salmonella-associated food poisoning.

\section{Materials and Methods}

\subsection{Sample Collection and Salmonella Isolation}

A total of 740,635 stool samples (approximately 60,000 samples per month) were collected from food workers in all 47 prefectures in Japan between January and December 2018. The food workers included cooks and servers in restaurants and food factory workers. The individuals periodically (mostly monthly) collected their stool samples using transport swabs with a modified Cary-Blair transport medium (Eiken Chimical Co., Tokyo, Japan) and then submitted them to one of two laboratories (the Incorporated Foundation Tokyo Kenbikyo-in and the BML Food Science Solutions) during the study period. We were not provided with the precise number of individuals who submitted stool samples or the number of facilities involved in sampling. The individuals did not report symptoms of enteritis upon submission of stool samples. Each sample was tested in laboratories within $72 \mathrm{~h}$ upon arrival. A tip of each swab was streaked onto a modified SalmonellaShigella agar plate (Eiken Chemical) and incubated at $37^{\circ} \mathrm{C}$ for $24 \mathrm{~h}$. Putative Salmonella colonies were biochemically identified as described previously [23]. One strain per sample was suspended in $20 \%$ glycerol and stored at $-80{ }^{\circ} \mathrm{C}$ until ready for serotyping and antimicrobial susceptibility testing.

\subsection{Local Chicken Products and Salmonella Isolation}

A total of 235 local chicken products (liver, breast, thigh, neck skin, and minced meat) were collected from 25 retail stores and 6 chicken processing plants between January 2018 and October 2021. The products were transported under refrigeration to the National Institute of Health Sciences. Each sample was tested within $24 \mathrm{~h}$ after arrival at the laboratory. Twenty-five grams of the sample was mixed in $225 \mathrm{~mL}$ of buffered peptone water (Oxoid Ltd., Hampshire, UK) and incubated at $37^{\circ} \mathrm{C}$ for $18 \mathrm{~h}$ for pre-enrichment. After incubation, 0.1 and $1 \mathrm{~mL}$ of the culture was added to $10 \mathrm{~mL}$ of Rappaport-Vassiliadis broth (Oxoid) and $10 \mathrm{~mL}$ of Hajna tetrathionate broth (Eiken Chemical), respectively, then incubated at $42{ }^{\circ} \mathrm{C}$ for $20 \mathrm{~h}$. After incubation, each culture was streaked onto two selective isolation agar plates: xylose-lysine-deoxycholate agar (Oxoid) and CHROMagar ${ }^{\mathrm{TM}}$ Salmonella (CHROMagar, Paris, France) then incubated at $37^{\circ} \mathrm{C}$ for $24 \mathrm{~h}$. For the selective isolation from minced meat, Mannitol-Lysine-Crystal-Violet-Brilliant-Green agar (Nissui Pharmaceutical, Tokyo, Japan) was used in place of xylose-lysine-deoxycholate agar. Putative Salmonella colonies were biochemically identified as mentioned above. One strain per sample was suspended in $20 \%$ glycerol and stored at $-80{ }^{\circ} \mathrm{C}$ until ready for serotyping and antimicrobial susceptibility testing.

\subsection{Serotyping}

Salmonella strains were tested for somatic antigens by slide agglutination using $\mathrm{O}$ antisera (Denka Co., Tokyo, Japan, and SSI Diagnostica, Copenhagen, Denmark). Salmonella strains were further tested for flagella antigens by tube agglutination using $\mathrm{H}$ antisera (Denka). Serovars were determined based on the reaction between $\mathrm{O}$ and $\mathrm{H}$ group antigens according to the Kauffmann-White scheme [24]. Strains agglutinated with anti-O4 and anti-H:i serum but not anti-H:1 or anti-H:2 serum were confirmed as monophasic variants of $S$. Typhimurium using a previously reported polymerase chain reaction method [25]. 


\subsection{Antimicrobial Susceptibility Testing}

The susceptibility of the Salmonella isolates to 11 antimicrobial agents was determined by antimicrobial susceptibility testing. Minimum inhibitory concentrations were determined by a two-fold broth microdilution method in 96-well microtiter plates (Dry-plate 'Eiken', Eiken Chemical), as described in the standards of the Clinical and Laboratory Standards Institute (CLSI) [26]. The following antimicrobial agents were tested: ampicillin (range of antimicrobial dilution: 1 to $128 \mathrm{mg} / \mathrm{L}$ ), cefazolin (1 to $128 \mathrm{mg} / \mathrm{L}$ ), cefotaxime ( 0.5 to $64 \mathrm{mg} / \mathrm{L})$, streptomycin (1 to $128 \mathrm{mg} / \mathrm{L}$ ), gentamicin $(0.5$ to $64 \mathrm{mg} / \mathrm{L})$, kanamycin (1 to $128 \mathrm{mg} / \mathrm{L}$ ), tetracycline ( 0.5 to $64 \mathrm{mg} / \mathrm{L}$ ), nalidixic acid (1 to $128 \mathrm{mg} / \mathrm{L}$ ), ciprofloxacin (0.03 to $4 \mathrm{mg} / \mathrm{L})$, colistin $(0.12$ to $16 \mathrm{mg} / \mathrm{L})$, and chloramphenicol (1 to $128 \mathrm{mg} / \mathrm{L})$. The $E s-$ cherichia coli reference strain ATCC 25922 was used as a control. The plates were incubated for $24 \pm 2 \mathrm{~h}$ at $37^{\circ} \mathrm{C}$ under aerobic conditions. The resistance breakpoints were defined based on the CLSI standard [27] and the Report on the Japanese Veterinary Antimicrobial Resistance Monitoring System 2016-2017 [28].

\subsection{Determination of Antimicrobial Resistance Genes and Sequence Types Based on MLST in Cefotaxime-Resistant Salmonella Strains by WGS Analysis}

DNA was extracted from cefotaxime-resistant strains using DNeasy ${ }^{\circledR}$ UltraClean ${ }^{\circledR}$ Microbial Kit (Qiagen GmbH, Hilden, Germany). WGS analysis was performed as previously described [29]. Sequencing libraries for each strain were prepared using QIAseq FX Library Kit (Qiagen) to obtain pair-end sequences $(300 \mathrm{bp} \times 2)$ using the Illumina Miseq platform. Draft genome sequences were obtained by assembling the read sequences using A5 miseq [30]. The WGS data were analyzed using the Database of Pathogen Genomics and Epidemiology, GenEpid-J [31], and by using the ResFinder tool available from the Center for Genomic Epidemiology (http:/ / www.genomicepidemiology.org/, accessed on 21 July 2021). MLST was performed using nucleotide sequences of seven housekeeping genes (aroC, dnaN, hemD, hisD, purE, sucA, and thrA) according to protocols available on the MLST database (https://pubmlst.org/organisms/salmonella-spp/, accessed on 23 November 2021).

\subsection{Statistical Analysis}

All statistical analyses were performed using $\mathrm{R}$ version 4.1. Confidence intervals were determined using the exact binomial test. Differences between proportions were tested using Fisher's exact test, where $p$-values of $<0.05$ were considered statistically significant.

\section{Conclusions}

Salmonella was isolated from $0.079 \%(583 / 740,635)$ of stool samples obtained from food workers in Japan. The strains were serotyped into 76 serovars and 22 untypeable Salmonella isolates. The top 14 serovars together accounted for over $70 \%$ of human-derived strains. A majority of the top 14 serovars in human-derived strains are prevalent in Japanese broiler and laying hen flocks. Of the 583 Salmonella strains, 12 (2.1\%) were resistant to the TGC cefotaxime and harbored the same bla genes as those observed in local and imported chicken products. The characterization of Salmonella isolated from food workers showed that local and imported chicken products may represent the primary sources of TGCresistant Salmonella responsible for infection among food workers. Although the prevalence of TGC-resistant Salmonella in food workers is low, they may contaminate food served to consumers. Therefore, monitoring the prevalence and characteristics of Salmonella in food workers is necessary for the risk management of Salmonella-associated food poisoning.

Author Contributions: Conceptualization, Y.S.; methodology, Y.S., T.A.; investigation, Y.S., H.K., Y.B., Y.H., M.Y., T.I. (Tetsuya Ikeda); resources, H.K., Y.B., T.I. (Takeshi Ito), Y.H., M.Y., K.O. and Y.H.-K.; supervision, M.K., T.A., H.A.; writing—original draft preparation, Y.S.; writing-review and editing, T.A. All authors have read and agreed to the published version of the manuscript. 
Funding: This study was supported by a grant-in aid from the Ministry of Health, Labour and Welfare of Japan (19KA1005 and 21KA1004) and the Japan Agency for Medical Research and the Development Research Program on Emerging and Re-emerging Infectious Diseases (JP21fk0108103).

Institutional Review Board Statement: Not applicable.

Informed Consent Statement: Not applicable.

Data Availability Statement: Data are contained within the article.

Conflicts of Interest: The authors declare no conflict of interest.

\section{References}

1. Ao, T.T.; Feasey, N.A.; Gordon, M.A.; Keddy, K.H.; Angulo, F.J.; Crump, J.A. Global burden of invasive nontyphoidal Salmonella disease, 2010. Emerg. Infect. Dis. 2015, 21, 941-949. [CrossRef]

2. World Health Organization. Critically Important Antimicrobials for Human Medicine, 6th ed.; World Health Organization: Geneva, Switzerland, 2019.

3. Hohmann, E.L. Nontyphoidal salmonellosis. Clin. Infect. Dis. 2001, 32, 263-269.

4. Yoshikura, H. Declining Vibrio parahaemolyticus and Salmonella, increasing Campylobacter and persisting norovirus food poisonings: Inference derived from food poisoning statistics of Japan. Jpn. J. Infect. Dis. 2020, 73, 102-110. [CrossRef]

5. Mori, T.; Okamura, N.; Kishino, K.; Wada, S.; Zou, B.; Nanba, T.; Ito, T. Prevalence and antimicrobial resistance of Salmonella serotypes isolated from poultry meat in Japan. Food Saf. 2018, 6, 126-129. [CrossRef] [PubMed]

6. Saito, S.; Koori, Y.; Ohsaki, Y.; Osaka, S.; Oana, K.; Nagano, Y.; Arakawa, Y.; Nagano, N. Third-generation cephalosporin-resistant non-typhoidal Salmonella isolated from human feces in Japan. Jpn. J. Infect. Dis. 2017, 70, 301-304. [CrossRef]

7. Kutsuna, S.; Yonetani, S.; Araki, K.; Izumiya, H. A case of pediatric with acute enteritis sue to CTX-M-15 extended-spectrum ß-lactamese-producing Salmoenlla Blockley. Jpn. J. Antimbiot. 2016, 69, 343-346.

8. Hiki, M.; Usui, M.; Kojima, A.; Ozawa, M.; Ishii, Y.; Asai, T. Diversity of plasmid replicons encoding the bla $a_{\mathrm{CMY}-2}$ gene in broad-spectrum cephalosporin-resistant Escherichia coli from livestock animals in Japan. Foodborne Pathog. Dis. 2013, 10, $243-249$. [CrossRef] [PubMed]

9. Xu, H.; Zhang, W.; Zhang, K.; Zhang, Y.; Wang, Z.; Zhang, W.; Li, Y.; Li, Q. Characterization of Salmonella serotypes prevalent in asymptomatic people and patients. BMC Infect. Dis. 2021, 21, 632. [CrossRef] [PubMed]

10. Kariuki, S.; Mbae, C.; Puyvelde, S.V.; Onsare, R.; Kavai, S.; Wairimu, C.; Ngetich, R.; Clemens, J.; Dougan, G. High relatedness of invasive multi-drug resistant non-typhoidal Salmonella genotypes among patients and asymptomatic carriers in endemic informal settlements in Kenya. PLoS Negl. Trop. Dis. 2020, 14, e0008440. [CrossRef]

11. Sasaki, Y.; Murakami, M.; Maruyama, N.; Tsujiyama, Y.; Kusukawa, M.; Asai, T.; Yamada, Y. Risk factors for Salmonella prevalence in laying-hen farms in Japan. Epidemiol. Infect. 2012, 140, 982-990. [CrossRef]

12. Sasaki, Y.; Ikeda, A.; Ishikawa, K.; Murakami, M.; Kusukawa, M.; Asai, T.; Yamada, Y. Prevalence and antimicrobial susceptibility of Salmonella in Japanese broiler flocks. Epidemiol. Infect. 2012, 140, 2074-2081. [CrossRef] [PubMed]

13. Shimojima, Y.; Nishino, Y.; Fukui, R.; Kuroda, S.; Suzuki, J.; Sadamasu, K. Salmonella serovars isolated from retail meats in Tokyo, Japan and their antimicrobial susceptibility. Shokuhin Eiseigaku Zasshi J. Food Hyg. Soc. Jpn. 2020, 61, 211-217. [CrossRef]

14. Ministry of Agriculture, Forestry and Fisheries of Japan. The 94th Statistical Yearbook of Ministry of Agriculture, Forestry and Fisheries; Ministry of Agriculture, Forestry and Fisheries: Tokyo, Japan, 2020.

15. Kijima, M.; Shirakawa, T.; Uchiyama, M.; Kawanishi, M.; Ozawa, M.; Koike, R. Trends in the serovar and antimicrobial resistance in clinical isolates of Salmonella enterica from cattle and pigs between 2002 and 2016 in Japan. J. Appl. Microbiol. 2019, 127, $1869-1875$. [CrossRef]

16. Shigemura, H.; Matsui, M.; Sekizuka, T.; Onozuka, D.; Noda, T.; Yamashita, A.; Kuroda, M.; Suzuki, S.; Kimura, H.; Fujimoto, S.; et al. Decrease in the prevalence of extended-spectrum cephalosporin-resistant Salmonella following cessation of ceftiofur use by the Japanese poultry industry. Int. J. Food. Microbiol. 2018, 274, 45-51. [CrossRef] [PubMed]

17. Voss-Rech, D.; Vaz, C.S.; Alves, L.; Coldebella, A.; Leão, J.A.; Rodrigues, D.P.; Back, A. A temporal study of Salmonella enterica serotypes from broiler farms in Brazil. Poult. Sci. 2015, 94, 433-441. [CrossRef] [PubMed]

18. Souza, A.I.S.; Saraiva, M.M.S.; Casas, M.R.T.; Oliveira, G.M.; Cardozo, M.V.; Benevides, V.P.; Barbosa, F.O.; Freitas Neto, O.C.; Almeida, A.M.; Berchieri, A., Jr. High occurrence of beta-lactamase-producing Salmonella Heidelberg from poultry origin. PLoS ONE 2020, 15, e0230676. [CrossRef]

19. Gelinski, J.M.; Bombassaro, A.; Baratto, C.M.; Vicente, V.A. Resistance to extended-spectrum beta-lactamases in Salmonella from a broiler supply chain. Int. J. Environ. Res. Public Health 2014, 11, 11718-11726. [CrossRef] [PubMed]

20. Dos Santos Bersot, L.; Carbonera, N.R.; Rodrigues Valcanaia, C.D.; Viana, C.; Nero, L.A. Multidrug-resistant and extendedspectrum beta-lactamase-producing Salmonella enterica serotype Heidelberg is widespread in a poultry processing facility in southern Brazil. J. Food Prot. 2021, 84, 2053-2058. [CrossRef]

21. Perin, A.P.; Martins, B.T.F.; Barreiros, M.A.B.; Yamatogi, R.S.; Nero, L.A.; Dos Santos Bersot, L. Occurrence, quantification, pulse types, and antimicrobial susceptibility of Salmonella sp. isolated from chicken meat in the State of Parana, Brazil. Braz. J. Microbiol. 2020, 51, 335-345. [CrossRef] 
22. Shigemura, H.; Sakatsume, E.; Sekizuka, T.; Yokoyama, H.; Hamada, K.; Etoh, Y.; Carle, Y.; Mizumoto, S.; Hirai, S.; Matsui, M.; et al. Food workers as a reservoir of extended-spectrum cephalosporin-resistant Salmonella strains in Japan. Appl. Environ. Microbiol. 2020, 86, e00072-20. [CrossRef]

23. Murakami, K.; Horikawa, K.; Ito, K.; Otsuki, K. Environmental survey of Salmonella and comparison of genotypic character with human isolates in Western Japan. Epidemiol. Infect. 2001, 126, 159-171. [CrossRef]

24. Grimont, P.A.D.; Weil, F. Antigenic Formulas of the Salmonella Serovars, 9th ed.; WHO Collaborating Centre for Reference and Research on Salmonella: Paris, France; Institute Pasteur: Paris, France, 2007.

25. Echeita, M.A.; Herrera, S.; Usera, M.A. Atypical, fljB-negative Salmonella enterica subsp. enterica strain of serovar 4,5,12:i: Appears to be a monophasic variant of serovar Typhimurium. J. Clin. Microbiol. 2001, 39, 2981-2983. [CrossRef]

26. Clinical and Laboratory Standards Institute. Performance Standards for Antimicrobial Disk and Dilution Susceptibility Tests for Bacteria Isolated from Animals, 4th ed.; VET08; CLSI: Wayne, PA, USA, 2018.

27. Clinical and Laboratory Standards Institute. Performance Standards for Antimicrobial Susceptibility Testing, 28th ed.; CLSI: Wayne, PA, USA, 2018; Volume M100.

28. National Veterinary Assay Laboratory of Japan. Report on the Japanese Veterinary Antimicrobial Resistance Monitoring System 2016-2017; National Veterinary Assay Laboratory, Ministry of Agriculture, Forestry and Fisheries: Tokyo, Japan, 2020.

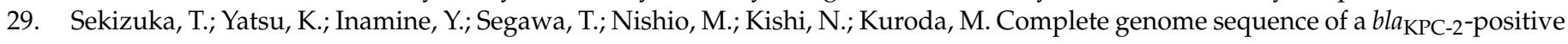
Klebsiella pneumoniae strain isolated from the effluent of an urban sewage treatment plant in Japan. mSphere 2018, 3, e00314-18. [PubMed]

30. Coil, D.; Jospin, G.; Darling, A.E. A5-miseq: An updated pipeline to assemble microbial genomes from Illumina MiSeq data. Bioinformatics 2015, 31, 587-589. [CrossRef] [PubMed]

31. Suzuki, S.; Ohnishi, M.; Kawanishi, M.; Akiba, M.; Kuroda, M. Investigation of a plasmid genome database for colistin-resistance gene mor-1. Lancet Infect. Dis. 2016, 16, 284-285. [PubMed] 